\title{
On total coloring of a Superposition snark family
}

\author{
Isabel F. A. Gonçalves, Simone Dantas, ${ }^{(1)}$ Diana Sasaki ${ }^{(1)}$
}

\begin{abstract}
The focus of this work is the total coloring and the equitable total coloring of snarks, a special class of cubic graphs. In 2003, Cavicchioli et al. [Acta Appl. Math. 76 (2003), pp. 56-88] asked about the existence of the smallest Type 2 snark with girth at least 5. In 2016, Dantas et al. [Discrete Appl. Math. 209 (2016), pp. 84-91] questioned the existence of a Type 1 cubic graph with girth at least 5 and equitable total chromatic number 5 . Recently, it has been proven that all Blowup snarks have equitable total chromatic number equal to 4 and in this paper we provide negative evidence to the question of Cavicchioli et al., proving that all members of an infinite family of snarks obtained by the Superposition operation of the Petersen graph and all Blowup snarks are Type 1.
\end{abstract}

2000 AMS Subject Classification: 05C15.

Keywords and Phrases: Equitable total coloring, total coloring, Blowup snarks, Superposition.

This study was financed in part by the Coordenação de Aperfeiçoamento de Pessoal de Nível Superior - Brasil (CAPES) - Finance Code 001, CAPES-PrInt project number 88881.310248/2018-01, CNPq and FAPERJ. 


\section{Introduction}

Let $G=(V, E)$ be a simple connected graph with vertex set $V$ and edges set $E$. A $k$-total coloring of $G$ is an assignment of $k$ colors to the edges and vertices of $G$, so that adjacent or incident elements have different colors. The smallest $k$ for which $G$ has a $k$-total coloring is the total chromatic number of $G$, denoted by $\chi^{\prime \prime}$. The acknowledged Total Color Conjecture [1, 16] states that $\Delta+1 \leq \chi^{\prime \prime} \leq \Delta+2$, where $\Delta$ is the maximum degree of $G$. A graph is said to be cubic if all its vertices have degree 3. Rosenfeld [13] and Vijayaditya [15] proved independently the Total Color Conjecture for cubic graphs, that is, the total chromatic number of a cubic graph is either 4 or 5 . Cubic graphs with $\chi^{\prime \prime}=4$ are said to be Type 1, and cubic graphs with $\chi^{\prime \prime}=5$ are said to be Type 2. If the difference between the cardinalities of any two color classes in a total coloring is at most one, then the coloring is said to be equitable. The equitable total chromatic number of $G$ is the smallest number for which $G$ admits an equitable total coloring and it is denoted by $\chi_{e}^{\prime \prime}$. Similarly to total coloring, Wang [17] conjectured that $\Delta+1 \leq \chi_{e}^{\prime \prime} \leq \Delta+2$.

In this paper, we focus on the total coloring of Snarks: bridgeless cubic graphs with chromatic number 4 . Their study was motivated by the search for counterexamples to the Four Color Conjecture. Gardner [6] named these graphs after the poem "The Hunting of the Snark" by Lewis Carroll. The importance of Snarks arises also from the fact that several well-known conjectures would have snarks as minimal counterexamples, as shown by Brinkmann et al. [2].

The girth of $G$ is the length of the shortest cycle contained in $G$. In 2003, Cavicchioli et al. 4] reported that all snarks with girth at least 5 and fewer than 30 vertices are Type 1 . In the same paper, they proposed the question of finding the smallest Type 2 snark with girth at least 5 . In 2011, Brinkmann et al. 2] extended this search and showed that all snarks with such girth and fewer than 38 vertices are Type 1. In 2014, Sasaki et al. 14 presented the first Type 2 snarks with small girth obtained 
from Type 1 snarks and verified that all members of Blanuša [18] and first Loupekine [11] infinite families are Type 1. Campos, Dantas, and de Mello [3] proved that all members of the infinite families of Flower 10] and Goldberg [7] snarks admit equitable 4-total colorings, so they are Type 1. In 2016, Dantas et al. [5] proved that the problem of determining the equitable total chromatic number of a cubic bipartite graph is NPcomplete and proposed the following question: Does there exist a Type 1 cubic graph with girth greater than 4 and equitable total chromatic number 5 ?

In this paper, we use our recent result on equitable total coloring of Blowup snarks [8] (Section 2), to provide evidence of a negative answer for Cavicchioli et al.'s question [4], that is that there is no Type 2 snark with girth at least 5 , by determining 4 -total colorings for all members of an infinite family obtained by the Superposition method, introduced by Kochol in 1996 [12], of the well known Petersen graph and all Blowup snarks (Section 3).

\section{Blowup snarks}

In this section, we present the construction of Blowup snarks and the proof about their equitable total chromatic number.

A semi-graph is a 3-tuple $G=(V, E, S)$ where $V$ is a finite set of vertices of $G, E$ is a set of edges having two distinct endpoints in $V$, and $S$ is a multiset of semi-edges having at most one endpoint in $V$. A semi-edge without endpoints is called an isolated edge. A semi-edge with endpoint $v$ is denoted by $v$. and an edge having endpoints $v$ and $w$ is denoted by $v w$. The definitions for simple graphs can be naturally extended to semigraphs. Indeed, a graph $G=(V, E)$ is a semi-graph with an empty set of semi-edges.

The infinite families considered in this paper are constructed by connecting semi-graphs through the junction of semi-edges. Given two semi-edges $v \cdot$ and $w \cdot$, the junction of $v \cdot$ and $w$. is done by replacing $v \cdot$ and $w \cdot$ by the 
edge $v w$. The construction of the equitable 4-total colorings consists of finding compatible 4-total colorings of the semi-graphs used in the construction of the family, so the obtained snark admits the desired equitable 4-total coloring.

The Blowup family of snarks was introduced in 2016 by Hägglund [9] and they are constructed by connecting copies of the semi-graph $D_{i}$ with vertex set $\left\{a_{i}, b_{i}, \ldots, l_{i}\right\}$, and six semi-edges depicted in Figure 1. More precisely, the $n$-Blowup with $n \geq 5$ is constructed through junctions: for $2 \leq i \leq n$, we make the junctions of semi-edges $a_{i} \cdot$ with $d_{i-1} \cdot, j_{i} \cdot$ with $k_{i-1} \cdot, l_{i} \cdot$ with $l_{i-1} \cdot ;$ and for $i=1, a_{1} \cdot$ with $d_{n} \cdot, j_{1} \cdot$ with $k_{n} \cdot$, and $l_{1} \cdot$ with $l_{n}$. We connect $n$ copies of $D_{i}$ similarly to a cycle, as shown in Figure 4 which represents the smallest cases covered by Theorem 2.1 the 5-Blowup and the 6-Blowup.

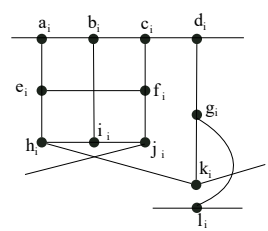

Figure 1: The semi-graph $D_{i}$.

Theorem 2.1 (Gonçalves et al. [8]). For $n \geq 5$, all $n$-Blowup have $\chi_{e}^{\prime \prime}=4$.

Proof. The construction of the coloring follows the construction of the family. Since we present colorings for fragments of an Blowup snark, we denote for $S_{k}, 2 \leq k \leq 4$, the semi-graph obtained by the junction of $k$ copies of the semi-graph $D_{i}$. We denote by $\varphi_{S_{k}}$ the equitable 4-total coloring of semi-graph $S_{k}$, and by $\varphi_{S_{k}}(a)$ the corresponding cardinality of the color class $a$ in $\varphi_{S_{k}}$. The semi-graphs $S_{2}$ and $S_{3}$ and their respective equitable 4-total colorings are shown in Figures 2 and 3.

Due to the junction that occurs in the construction, when determining the cardinality of a color in a semi-graph, we count each semi-edge as 0.5. 
Following this rule, we have that $\varphi_{S_{2}}(1)=\varphi_{S_{2}}(2)=\varphi_{S_{2}}(3)=\varphi_{S_{2}}(4)=15$, $\varphi_{S_{3}}(1)=\varphi_{S_{3}}(4)=22$ and $\varphi_{S_{3}}(2)=\varphi_{S_{3}}(3)=23$.

Let $\psi_{n}$ be the equitable 4 -total coloring of an $n$-Blowup, and $\psi_{n}(a)$ be the corresponding cardinality of color class $a$ in $\psi_{n}$.

For every $n$-Blowup with $n \equiv 0(\bmod 2)$, we construct an equitable 4-total coloring $\psi_{n}$ by repeating coloring $\varphi_{S_{2}}$ for $\frac{n}{2}$ copies of semi-graph $S_{2}$. We observe that the coloring fits the junctions used in construction. So, $\psi_{n}(1)=\psi_{n}(2)=\psi_{n}(3)=\psi_{n}(4)=\frac{n}{2} \times 15$.

For every $n$-Blowup with $n \equiv 1(\bmod 2)$, we construct an equitable 4total coloring $\psi_{n}$ by repeating coloring $\varphi_{S_{2}}$ for $\frac{n-3}{2}$ copies of semi-graph $S_{2}$, and $\varphi_{S_{3}}$ for one copy of semi-graph $S_{3}$. So, $\psi_{n}(1)=\psi_{n}(4)=\frac{n-3}{2} \times 15+22$, and $\psi_{n}(2)=\psi_{n}(3)=\frac{n-3}{2} \times 15+23$. Thus, $\psi_{n}(1), \psi_{n}(2), \psi_{n}(3)$, and $\psi_{n}(4)$ differ by at most one and thus the obtained 4 -total colorings are equitable.

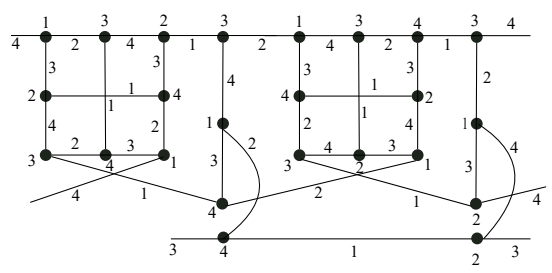

Figure 2: The semi-graph $S_{2}$ with equitable 4-total coloring $\varphi_{S_{2}}$.

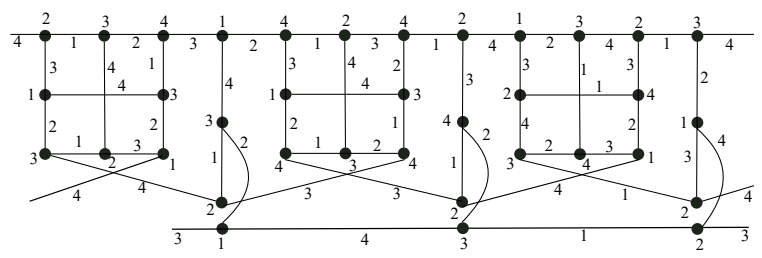

Figure 3: The semi-graph $S_{3}$ with equitable 4-total coloring $\varphi_{S_{3}}$. 

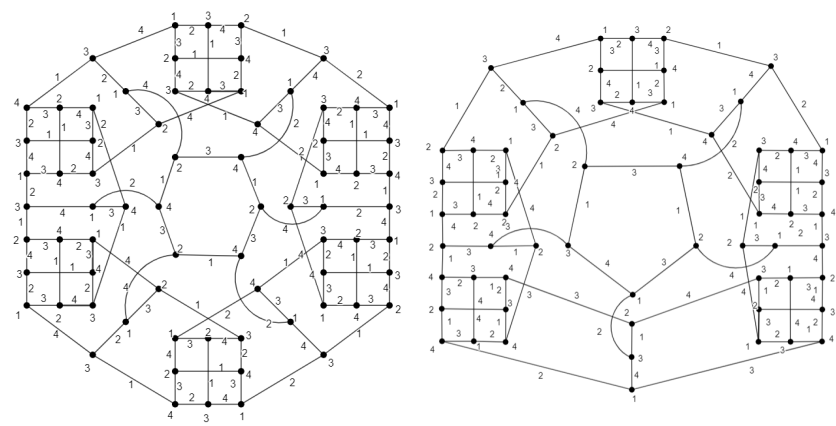

Figure 4: Snarks 6-Blowup (left) and 5-Blowup (right) with their equitable 4-total colorings $\psi_{6}$ and $\psi_{5}$, respectively.

\section{Superposition snarks}

In this section, we construct an infinite family of snarks obtained through the Superposition construction introduced by Kochol in 1996 [12. With this method, Kochol was able to construct snarks with large girth, and this seems an approach to the search of a Type 2 snark with girth at least 5 . However, in the case of the next considered family, all members are Type 1.

Given a cubic semi-graph $M(V, E, S)$, the set $S$ of semi-edges is denoted by $S(M)$ and is partitioned into $n$ pairwise disjoint nonempty sets $H_{1}, H_{2}$, $\ldots, H_{n}$ such that $\left|H_{i}\right|=k_{i}$ with $i=1,2, \ldots, n$ and $\sum_{i=1}^{n} k_{i}=|S(M)|$. Following Kochol's notation [12], we call the sets $H_{i}$ connectors and denote the semi-graph $M$ by $\left(k_{1}, k_{2}, \ldots, k_{n}\right)$-semi-graph $M$. A superedge is a semi-graph with two connectors, and a supervertex is a semi-graph with three connectors. Now, we consider the following semi-graphs depicted in Figure 5:

- (3,3)-semi-graph $G^{\prime}$ (superedge) is obtained by removing two nonadjacent vertices $v_{1}$ and $v_{2}$ of a snark $G$; Note that the remaining semi-edges can form or be part of a connector. 
- $(1,1)$-semi-graph $L^{\prime}$ (superedge) is an isolated edge (two semi-edges);

- $(1,3,3)$-semi-graph $J^{\prime}$ (supervertex) consists of two isolated edges and a vertex;

- $(1,1,1)$-semi-graph $K^{\prime}$ (supervertex) consists of a vertex and three semi-edges.

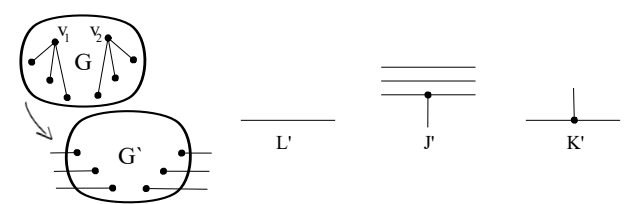

Figure 5: Superedge $G^{\prime}$, superedge $L^{\prime}$, supervertex $J^{\prime}$ and supervertex $K^{\prime}$.

Let $G$ be a snark. Replace every edge $e \in E$ by a superedge $\xi$ and every vertex $v \in V$ by a supervertex $\vartheta$. If $v$ is incident to $e$ then a connector in $\vartheta_{v}$ will be linked with a connector in $\xi_{e}$ through the junction of semiedges. The cubic graph obtained is called Superposition of $G$ denoted by $G(\xi, \vartheta)$ and it is a snark [12]. Next, we introduce an application of the Superposition construction that generates a new family of snarks which are Type 1.

Theorem 3.1. All snarks obtained by a Superposition of the Petersen graph and an $n$-Blowup snark with $n \geq 6$ have $\chi^{\prime \prime}=4$.

Proof. In order to obtain 4-total colorings for the resulting snarks, we use specific colored superedges $C_{1}, C_{2}, C_{3}, C_{4}$ obtained by removing two nonadjacent vertices from the $n$-Blowup. More precisely, we choose two specific vertices from the semi-graph $S_{4}$, which equitable 4 -total coloring is obtained by using twice the coloring for $S_{2}$ of Figure 2. Note that this semi-graph appears in every $n$-Blowup with $n \geq 6$. Thus, to obtain the superedges $C_{1}, \ldots, C_{4}$, we remove the respectively 4 pairs of nonadjacent vertices: $\left\{h_{1}, k_{3}\right\},\left\{b_{2}, f_{1}\right\},\left\{b_{2}, e_{2}\right\}$ and $\left\{c_{1}, k_{1}\right\}$. 
Now, consider the Petersen graph depicted with a convenient representation in Figure 6. For every $1 \leq i \leq 6$, replace the vertex $x_{i}$ for the supervertex $J^{\prime}$ depicted in Figure 5. Next, replace edges $x_{3} x_{4}$ and $x_{5} x_{6}$ by a copy of the superedge $C_{1}$, edges $x_{2} x_{3}$ and $x_{4} x_{5}$ by a copy of superedge $C_{2}$, edge $x_{6} x_{1}$ by a copy of superedge $C_{3}$ and edge $x_{1} x_{2}$ by a copy of superedge $C_{4}$. The remaining edges (resp. vertex) are replaced by superedge $L^{\prime}$ (resp. supervertex $K^{\prime}$ ). We observe that replacing an edge (resp. vertex) by superedge $L^{\prime}$ (resp. supervertex $K^{\prime}$ ) is equivalent to maintain the original edge (resp. vertex). Figure 6 presents a depiction of the obtained graph with its 4 -total coloring.

Let $\phi_{n}$ be the 4-total coloring of the snark generated from the superposition of a Petersen graph and an $n$-blowup snark and $\phi_{n}(a)$ be the corresponding cardinality of color class $a$ in $\phi_{n}$. So, if $n \equiv 0(\bmod 2)$, $\phi_{n}(1)=11+45 n, \phi_{n}(2)=10+45 n, \phi_{n}(3)=\phi_{n}(4)=9+45 n$. If $n \equiv 1(\bmod 2), \phi_{n}(1)=\frac{45}{2}(n-3)+73, \phi_{n}(2)=\frac{45}{2}(n-3)+75$, $\phi_{n}(3)=\frac{45}{2}(n-3)+72$ and $\phi_{n}(4)=\frac{45}{2}(n-3)+69$. Therefore, for every $n \geq 6$, the obtained 4 -total-coloring is not equitable.

Note that we obtain an infinite family, since each $n$-Blowup with $n \geq 6$ generates a new snark with $66 n+10$ vertices. Indeed, the first member of the family, obtained from the 6-Blowup, has 406 vertices. Moreover, considering that the removed two vertices belong to semi-graphs such that their remaining elements (edges and vertices) are preserved in every $n$ Blowup, it is easy to verify that every obtained superedge $C_{i}, i=1, \ldots, 4$, matches the coloring depicted in Figure 6, no matter which $n$-Blowup it was build from. So the 4-total colorings constructed in Theorem 2.1 can be naturally used in every superedge $C_{i}$; and each member of the new family admits a 4-total coloring which is an extension of the total coloring presented in Figure6. 


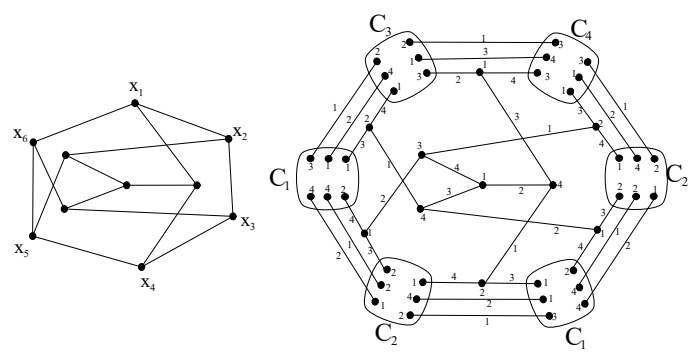

Figure 6: The Petersen graph (left) and a depiction of the 4-total colored Superposition construction (right).

\section{References}

[1] M. Behzad, Graphs and their chromatic numbers, Ph.D. Thesis, Michigan State University, East Lansing, MI, USA, 1965.

[2] G. Brinkmann, J. Jan Goedgebeur, J. Jonas Hägglund, K. Klas Markström, Generation and properties of snarks, J. Combin. Theory Ser. B 103, 4, 468-488, 2013.

[3] C. N. Campos, S. Dantas, C. P. De Mello, The total-chromatic number of some families of snarks, Discrete Math., 311, 984-988, 2011.

[4] A. Cavicchioli, T. E. Murgolo, B. Ruini, F. Spaggiari, Special classes of snarks, Acta Appl. Math., 76, 56-88, 2003.

[5] S. Dantas, C. M. H. De Figueiredo, G. Mazzuoccolo, M. Preissmann, V. F. dos Santos, D. Sasaki, On the equitable total chromatic number of cubic graphs, Discrete Appl. Math., 209, 84-91, 2016.

[6] M. Gardner, Mathematical games: Snarks, boojums and other conjectures related to the fourcolor-map theorem, Sci. Am. 234, 126-130, 1976.

[7] M. K. Goldberg, Construction of class 2 graphs with maximum vertex degree 3, J. Combin. Theory Ser. B, 31, 282-291, 1981. 
[8] I. F. A. Gonçalves, S. Dantas and D. Sasaki, On equitable total coloring of snarks. In: XI LAGOS. Latin-American Algorithms, Graphs and Optimization Symposium, 2021, São Paulo. Electronic Notes in Theoretical Computer Science, to appear.

[9] J. Hägglund, On Snarks that are far from beeing 3-edge-colorable, Electron. J. Comb., 3, 23, 2016.

[10] R. Isaacs, Infinite Families of Nontrivial Trivalent Graphs Which Are Not Tait Colorable, Amer. Math. Monthly. 82, 221-239, 1975.

[11] R. Isaacs, Loupekine's snarks: a bifamily of non Tait colorable graphs, Technical Report 263, Dpt. of Math. Sci., The Johns Hopkins University, Maryland, USA, 1976.

[12] M. Kochol, Snarks without small cycles, J. Combin. Theory Ser. B, 67, 34-47, 1996.

[13] M. Rosenfeld, On the total coloring of certain graphs, Isr. J. Math, 9, 396-402, 1971.

[14] D. Sasaki, S. Dantas, C. M. H. De Figueiredo, M. Preissmann, The hunting of a snark with total chromatic number 5, Discrete Appl. Math., 164, 470-481, 2014.

[15] N. Vijayaditya, On total chromatic number of a graph, J. London Math. Soc., 3(2), 405-408, 1971.

[16] V. G. Vizing, On an estimate of the chromatic class of a p-graph, Diskret, Analiz, 3, 25-30, 1964.

[17] W. F. Wang, Equitable total coloring of graphs with maximum degree, Graphs Combin., 18, 677-685, 2002.

[18] J.J. Watkins, Snarks, Ann. New York Acad. Sci., 576, 606-622, 1989. 
Isabel F. A. Gonçalves

Fluminense Federal University

Institute of Mathematics and

Statistics, Brazil

isabelfigueira@id.uff.br
Simone Dantas

Fluminense Federal University

Institute of Mathematics and Statistics, Brazil

sdantas@id.uff.br

\section{Diana Sasaki}

Rio de Janeiro State University

Institute of Mathematics and

Statistics, Brazil

diana.sasaki@ime.uerj.br 